\title{
An updated review of mucosal melanoma: Survival meta-analysis
}

\author{
HYUNG MIN HAHN ${ }^{1}$, KYOUNG GEUN LEE ${ }^{2}$, WON CHOI ${ }^{2}$, SEUNG HYUN CHEONG ${ }^{2}$, \\ KI BUM MYUNG ${ }^{2}$ and HYUNG JIN HAHN ${ }^{2,3}$
}

\author{
${ }^{1}$ Department of Plastic and Reconstructive Surgery, Ajou University School of Medicine, Suwon, Gyeonggi 16499; \\ ${ }^{2}$ Department of Dermatology, College of Medicine; ${ }^{3}$ Myunggok Medical Research Institute, College of Medicine, \\ Konyang University, Daejeon, Chungcheongnam 35365, Republic of Korea
}

Received August 6, 2018; Accepted April 10, 2019

DOI: $10.3892 / \mathrm{mco} .2019 .1870$

\begin{abstract}
Mucosal melanoma (MM) is a highly lethal variant of melanoma that carries a poor prognosis. Extremely low incidence and survival rates have led to few clinical trials, and a lack of protocols and guidelines. The present study performed a survival meta-analysis for the quantitative synthesis of available evidence to search for key patterns that would help clinicians tailor optimal therapeutic strategies in MM. PubMed, EMBASE, Cochrane, MEDLINE, Google Scholar and other databases were searched. Hazard ratios, in disease-specific and overall survival, were calculated for each of the survival-determining variables. MM was 2.25 times more lethal than cutaneous melanoma (CM). The most significant threats to survival were advanced Tumor-Node-Metastasis stage, sino-nasal location, and old age. Chemotherapy was the most effective form of adjuvant therapy. Disease-specific survival, the primary measure of the effect sizes, can fluctuate depending on the accuracy of the reported cause of mortality. In conclusion, MM is a peculiar type of melanoma, with clinical and molecular profile vastly different from the much-familiar $\mathrm{CM}$. In the wake of the era of precision oncology, further
\end{abstract}

Correspondence to: Professor Hyung Jin Hahn, Department of Dermatology, College of Medicine, Konyang University, 158 Gwanjeodong-ro, Seo-gu, Daejeon, Chungcheongnam 35365, Republic of Korea

E-mail: clemens272@gmail.com

Abbreviations: AJCC, American Joint Committee on Cancer; ALM, acral lentiginous melanoma; CI, confidence interval; CM, cutaneous melanoma; DSS, disease-specific survival; ES, effect sizes; H\&N, head and neck; HR, hazard ratio; I2, degree of inconsistency; LDH, lactate dehydrogenase; LVI, lympho-vascular invasion; LRC, loco-regional control; MM, mucosal melanoma; MMHN, mucosal melanoma of head and neck; MSS, melanoma-specific survival; NR, not reported; OC, oral cavity; OS, overall survival; PNI, perineural invasion; PNS, paranasal sinuses; PS, performance score; RCT, randomised controlled trials; RT, radiotherapy; RTK, receptor tyrosine kinase; SN, sino-nasal; UV, ultraviolet

Key words: survival meta-analysis, HR, MM, disease-specific survival, OS studies on driver mutations and oncogenic pathways would likely lead to improved patient survival.

\section{Introduction}

Mucosal melanoma (MM) represents a highly aggressive variant of malignant melanoma that arises within the resident melanocytes of mucous linings. Comprising barely one-hundredth fraction of all melanomas, it is an entity that is notorious for the infinitesimal 5-year survival rate $(<25 \%)(1)$. Although MM is often understood as a blanket term for any extracutaneous melanoma, it nevertheless comes with somewhat hazy disease definition; some authors regard uveal or conjunctival melanomas as bona fide MM, while others are less inclined to label the ocular tumours as such. The head and neck $(\mathrm{H} \& \mathrm{~N})$ is cited as the region most heavily represented $(\sim 50 \%)$, followed by the ano-rectum, and the female genital tract (FGT) (2). The insidious nature of the disease compounds accurate diagnosis, depriving the affected of any remaining chance for an early detection. Failure to intervene early often boomerangs with the amplified lethality, which is the hallmark of the mucosal disease.

Given the miniscule incidence and patient survival rate, randomised clinical trials (RCT) have been understandably difficult to come by. The resulting paucity of evidence have long clouded our understanding of tumour behaviour. Field clinicians facing therapeutic decisions inevitably suffer from general lack of consensus over virtually all aspects of the disease, from staging to management. While it is tempting to extrapolate from CM-derived data, the notion, that $\mathrm{MM}$ is fundamentally a distinctive entity, is now considered canonical (3). Such discrepancies include female preponderance, limited role of UV (ultraviolet) light, and mutation status (4). The different makeup of mutation landscape is thought to be the impetus that drives the divergence between the two (5-7).

In the present meta-analysis and systematic review, the authors present a comprehensive assessment of available evidence to elaborate crucial factors that determine clinical outcome in MM.

\section{Materials and methods}

Data collection and inclusion criteria. Literature search was conducted using multiple engines, most notably but not 


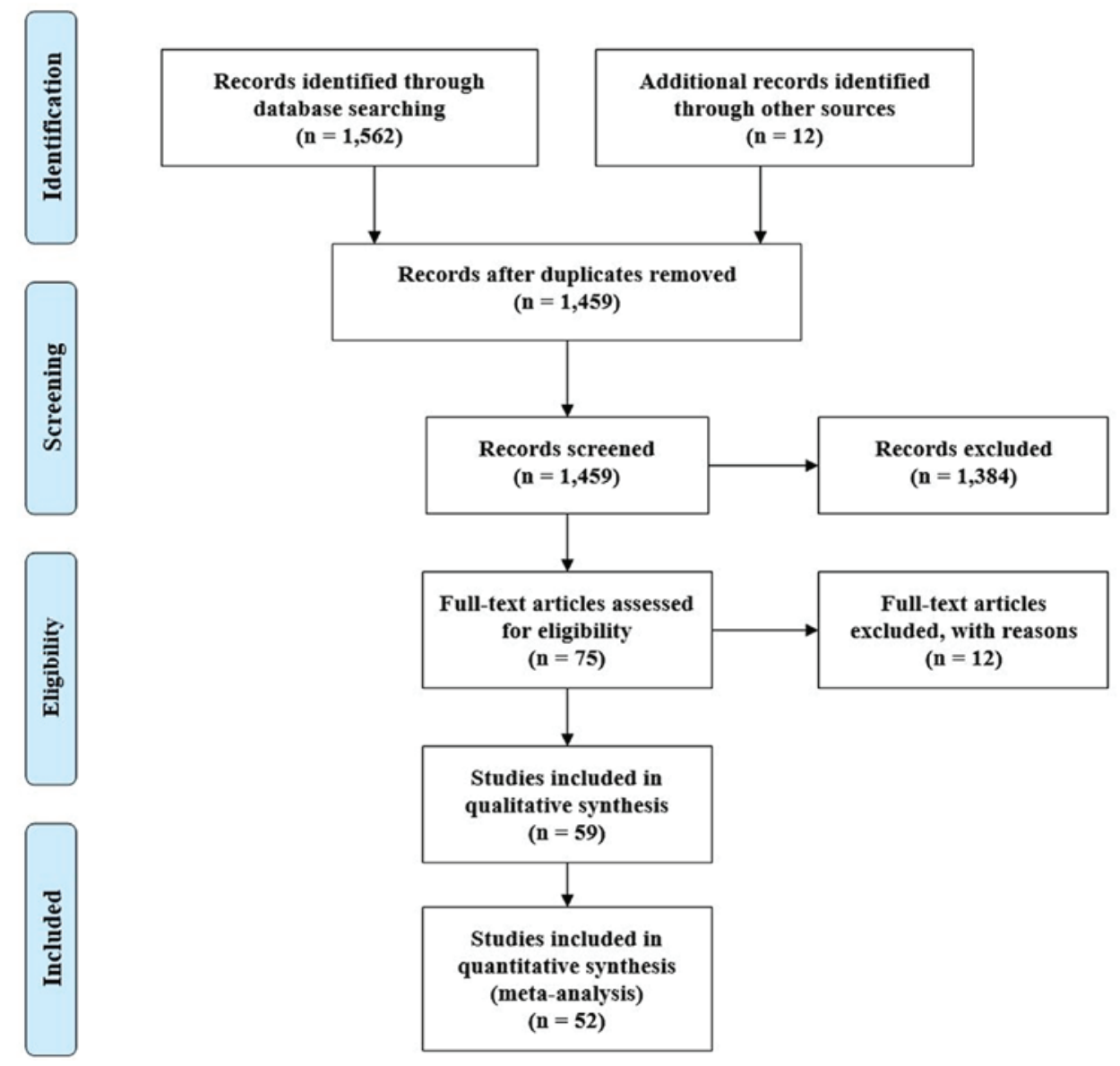

Figure 1. Flowchart of search strategy, adopted from the PRISMA Group, 2009 (10).

limited to, PubMed, EMBASE, Cochrane, MEDLINE, and Google Scholar, up to March of 2018. The query employed various keywords, such as 'mucosal malignant melanom', 'anorectal melanoma', 'sino-nasal melanoma' and 'survival'; the search was intended to include any abstract proceedings or graduate theses [www.thesis.de], so as not to discount 'grey' literature from the study. No restriction was applied in terms of the language of publication. The following criteria were considered for selection: i) primary mucosal melanomas, ii) reporting of Kaplan-Meier survival analysis results, or iii) Cox regression analysis with time-to-event information. Where HR were not explicitly given, they were imputed using the method described by Tierney et al (8). Excluded were studies i) on leptomeningeal melanomatosis, ii) based on cell lines iii) performed on canine, murine or other non-human subjects. The present study was conducted in accordance to the Meta-analysis of Observational Studies in Epidemiology guidelines for the reporting of meta-analyses of observational studies (MOOSE) (9).

Statistical analysis. The principal parameter of effect size (ES) reporting used in the study was hazard ratio (HR), in terms of melanoma-specific survival (=disease-specific survival, DSS) and overall survival (=all-cause survival, OS). The main surrogate for detecting between-study heterogeneity was the $\mathrm{I}^{2}$ statistic. The assumption of homogeneity was considered valid if $\mathrm{I}^{2}$ was $<50 \%$, in which cases the fixed effect model was used; for all other cases, the random effect model was used. Before incorporating a study into analysis, sensitivity testing was performed to decide if there was a pulling effect by single studies with substantial weight. Publication bias was assessed with funnel plots and Egger test. Statistical analyses were carried out with Comprehensive Meta-Analysis Software (v3.0; Biostat, Englewood, NJ, USA). P<0.05 were considered to indicate a statistically significant difference.

\section{Results}

PRISMA (Preferred Reporting Items for Systematic Reviews and Meta-Analyses) (10) flow diagram of the search strategy, and characteristics of the included studies are given in Fig. 1 and Table I, respectively. Search query using the aforementioned keywords initially returned 1,459 articles from 8 different databases, of which 52 were deemed to suit our agenda. All the studies originated from three continent regions: North/Central America (18, 34.6\%), Asia/Indian subcontinent/Oceania $(21,40.4 \%)$, and the European Union $(13,25.0 \%)$. Topographically, 27 studies $(51.9 \%)$ were on head and neck region $(\mathrm{MMHN}), 4(7.7 \%)$ on gastrointestinal tract, $3(5.8 \%)$ on urinary/female genital tract, and $18(34.6 \%)$ on all mucosal sites. Potential survival variables were arbitrarily categorised into three groups: i) host factors, which is demographic characteristics of the affected individual, ii) tumour factors, relating to various aspects of tumour histology, behaviour, and staging, and iii) treatment factors, which are parameters that assess the impact of differing treatment modalities on survival. 
Table I. Characteristics of included studies.

\begin{tabular}{|c|c|c|c|c|c|}
\hline Author, year & Country $^{\mathrm{b}}$ & Location & No. of patients & Follow-up & Ref. \\
\hline Abugideiri et al, 2016 & USA & $\mathrm{H} \& \mathrm{~N}$ & $39(\mathrm{SRT}=27 ; \mathrm{S}=12)$ & Median 8.1 years & 17 \\
\hline Ahn et al, 2010 & Korea & $\mathrm{H} \& \mathrm{~N}$ & $32(\mathrm{SRT}=16 ; \mathrm{S}=16)$ & Median 25.3 months & 18 \\
\hline Aiempanakit et al, 2018 & Thailand & All mucosal & $17(\mathrm{~S}=14, \mathrm{UN}=3)$ & Median 18.2 months & 19 \\
\hline Ajmani et al, 2017 & USA & $\mathrm{SN}$ & $704(\mathrm{SRT}=399 ; \mathrm{S}=305)$ & NR & 20 \\
\hline Amit et al, 2018 & USA & SN & $\begin{array}{l}198(\mathrm{SRT}=81 ; \mathrm{S}=79 ; \\
\mathrm{SCRT}=24 ; \mathrm{C} \text { or } \mathrm{CRT}=14)\end{array}$ & Median 26 months & 21 \\
\hline D'Angelo et al, 2016 & USA & All mucosal & $\begin{array}{l}889 \text { (ipilimumab } \\
\text { and nivolumab) }\end{array}$ & 6.2 months & 22 \\
\hline Benlyazid et al, 2010 & France & $\mathrm{H} \& \mathrm{~N}$ & $160(\mathrm{SRT}=78 ; \mathrm{S}=82)$ & Median 65.2 months & 23 \\
\hline $\begin{array}{l}\text { Bishop and } \\
\text { Olszewski } 2014\end{array}$ & USA & All, including $\mathrm{CM}^{\mathrm{a}}$ & $229,976(\mathrm{NR})$ & NR & 24 \\
\hline $\begin{array}{l}\text { Chiu and } \\
\text { Weinstock, } 1996\end{array}$ & USA & $\mathrm{OC}$ & $40,320(\mathrm{NR})$ & NR & 25 \\
\hline Ciarrocchi et al, 2017 & Italy & Anorectum & $208(\mathrm{SRT}=32 ; \mathrm{S}=167)$ & Median 14 months & 26 \\
\hline Ercelep et al, 2016 & Turkey & All mucosal & $229,976(\mathrm{NR})$ & Median 27 months & 27 \\
\hline Harada et al, 2016 & Japan & Oesophagus & $10(\mathrm{~S}=10)$ & NR & 28 \\
\hline Hasebe et al, 2017 & Japan & $\mathrm{H} \& \mathrm{~N}$ & $85(\mathrm{RT}=85)$ & Median 42.5 months & 29 \\
\hline $\begin{array}{l}\text { Heinzelmann- } \\
\text { Schwarz et al, } 2014\end{array}$ & Australia & Vulva & 33 (NR) & NR & 30 \\
\hline Heppt et al, 2017 & Germany & All mucosal & 444 (NR) & NR & 31 \\
\hline Hughes et al, 2013 & Australia & All, including $\mathrm{CM}^{\mathrm{a}}$ & 485 (Lymphadenectomy) & Median 17.4 months & 32 \\
\hline Jang et al, 2014 & Korea & All, including $\mathrm{CM}^{\mathrm{a}}$ & $\begin{array}{l}206(S=197 ; C=46 \\
R T=31)\end{array}$ & $\mathrm{NR}$ & 33 \\
\hline Kang et al, 2018 & China & All mucosal & $60(\mathrm{NR})$ & Median 36 months & 34 \\
\hline Khan et al, 2014 & USA & $\mathrm{SN}$ & 567 (NR) & NR & 35 \\
\hline Kirchoff et al, 2016 & USA & All mucosal & $\begin{array}{l}227(S=53 \\
S+\text { other }=174)\end{array}$ & NR & 36 \\
\hline Kirschner et al, 2013 & USA & Vagina & $\begin{array}{l}201(\mathrm{SRT}=53 ; \\
\mathrm{S}=87 ; \mathrm{RT}=30)\end{array}$ & Median 14 months & 37 \\
\hline Kong et al, 2016 & China & All, including $\mathrm{CM}^{\mathrm{a}}$ & $412(\mathrm{NR})$ & Median 31 months & 38 \\
\hline Konuthula et al, 2017 & USA & SN & $\begin{array}{l}695(\mathrm{SRT}=271 ; \mathrm{S}=206 ; \\
\mathrm{SC}=29 ; \mathrm{SCRT}=49 \\
\mathrm{C}=21 ; \mathrm{RT}=42)\end{array}$ & NR & 39 \\
\hline Koto et al, 2017 & Japan & $\mathrm{H} \& \mathrm{~N}$ & $260(\mathrm{RT}=105 ; \mathrm{CRT}=155)$ & Median 22 months & 40 \\
\hline Kuk et al, 2016 & Korea & $\mathrm{OC}$ & $39(\mathrm{~S}=22 ; \mathrm{S}+\mathrm{C}$ or $\mathrm{RT}=17)$ & NR & 41 \\
\hline Lansu et al, 2018 & Netherlands & $\mathrm{SN}$ & $63(\mathrm{SRT}=63)$ & Median 23 months & 42 \\
\hline Lawaetz et al, 2016 & Denmark & $\mathrm{H} \& \mathrm{~N}$ & $\begin{array}{l}98(\mathrm{SRT}=26 ; \mathrm{S}=49 ; \mathrm{SC}=2 ; \\
\mathrm{SCRT}=2 ; \mathrm{RT}=8 ; \text { None }=8)\end{array}$ & Median 24.5 months & 43 \\
\hline Lee et al, 2017 & Korea & $\mathrm{H} \& \mathrm{~N}$ & $\begin{array}{l}31(\mathrm{SRT}=13 ; \mathrm{S}=9 \\
\mathrm{SC}=7 ; \mathrm{SCRT}=2)\end{array}$ & Mean 9 months & 44 \\
\hline Lee et al, 2017 & USA & $\mathrm{OC}$ & 232 (NR) & NR & 45 \\
\hline Lombardi et al, 2016 & Italy & $\mathrm{SN}$ & $58(\mathrm{SRT}=13 ; \mathrm{S}=42 ; \mathrm{SCRT}=3)$ & Median 30 months & 46 \\
\hline Mücke et al, 2009 & Germany & $\mathrm{OC}$ & $10(\mathrm{NR})$ & NR & 47 \\
\hline Nakamura et al, 2018 & Japan & All mucosal & 45 (checkpoint inhibitors) & NR & 48 \\
\hline Oba et al, 2011 & Japan & All, including $\mathrm{CM}^{\mathrm{a}}$ & $78(\mathrm{NR})$ & Median 40 months & 49 \\
\hline Pandey et al, 2002 & India & $\mathrm{H} \& \mathrm{~N}$ & $\begin{array}{l}60(\mathrm{SRT}=6 ; \mathrm{S}=17 ; \mathrm{SC}=3 ; \\
\mathrm{SCRT}=1 ; \mathrm{C}=8 ; \mathrm{RT}=7)\end{array}$ & NR & 50 \\
\hline Pfeil et al, 2011 & Germany & All mucosal & $172(\mathrm{NR})$ & Median 24 months & 51 \\
\hline Plave et al, 2016 & Slovenia & $\mathrm{H} \& \mathrm{~N}$ & $\begin{array}{l}61(\mathrm{SRT}=14 ; \mathrm{S}=17 ; \\
\mathrm{C}=1 ; \mathrm{RT}=15)\end{array}$ & Median 16.5 months & 52 \\
\hline Roh et al, 2016 & Korea & All mucosal & $392(\mathrm{NR})$ & Mean 55.4 months & 53 \\
\hline Samstein et al, 2016 & USA & $\mathrm{SN}$ & $78(\mathrm{SRT}=64 ; \mathrm{S}=14)$ & Median 21 months & 54 \\
\hline
\end{tabular}


Table I. Continued.

\begin{tabular}{|c|c|c|c|c|c|}
\hline Author, year & Country $^{\mathrm{b}}$ & Location & No. of patients & Follow-up & Ref. \\
\hline Sanchez et al, 2016 & USA & Genitourinary tract & $1,586(\mathrm{NR})$ & NR & 55 \\
\hline Schaefer et al, 2017 & Germany & All mucosal & 75 (checkpoint inhibitors) & $\mathrm{NR}$ & 56 \\
\hline Schmidt et al, 2017 & USA & $\mathrm{H} \& \mathrm{~N}$ & $\begin{array}{l}1,368(\mathrm{SRT}=704 \\
\mathrm{S}=566 ; \mathrm{RT}=98)\end{array}$ & Median 55.2 months & 57 \\
\hline Shoushtari et al, 2017 & USA & All mucosal & $81(\mathrm{NR})$ & NR & 58 \\
\hline Shuman et al, 2011 & USA & $\mathrm{H} \& \mathrm{~N}$ & $\begin{array}{l}52(\mathrm{SRT}=15 ; \mathrm{S}=13 ; \\
\mathrm{SC}=18 ; \mathrm{NR}=6)\end{array}$ & Median 97 months & 59 \\
\hline Song et al, 2016 & China & $\mathrm{OC}$ & $62(\mathrm{NR})$ & Median 32.5 months & 60 \\
\hline Sun et al, 2014 & China & $\mathrm{SN}$ & $\begin{array}{l}65(\mathrm{SRT}=13 ; \mathrm{S}=18 ; \mathrm{SC}=9 ; \\
\mathrm{C}=6 ; \mathrm{RT}=4 ; \mathrm{CRT}=2)\end{array}$ & NR & 61 \\
\hline Tchelebi et al, 2016 & USA & Rectum & $63(\mathrm{SRT}=18 ; \mathrm{S}=45)$ & Median 17 months & 62 \\
\hline Thariat et al, 2011 & France & $\mathrm{SN}$ & $155(\mathrm{NR})$ & Median 37 months & 63 \\
\hline Wang et al, 2013 & China & $\mathrm{OC}$ & $81(\mathrm{NR})$ & NR & 64 \\
\hline Wen et al, 2017 & China & All mucosal & $\begin{array}{l}52 \text { (checkpoint and } \\
\text { PD- } 1 \text { inhibitors) }\end{array}$ & NR & 65 \\
\hline Won et al, 2015 & Korea & SN & $155(\mathrm{NR})$ & NR & 66 \\
\hline Yeh et al, 2006 & USA & Anorectum & $46(S=23 ; C=23)$ & Median 29 months & 67 \\
\hline Yi et al, 2011 & Korea & All, including $\mathrm{CM}^{\mathrm{a}}$ & $95(\mathrm{NR})$ & Median 41 months & 68 \\
\hline
\end{tabular}

ancluded for purpose of comparison with mucosal melanoma; ${ }^{\text {}}$ For multi-national groups, only the nationality of 1 st author was listed. H\&N, head and neck; SN, sino-nasal; CM, cutaneous melanoma; OC, oral cavity; S, surgery only; C, chemotherapy only; RT, radiotherapy only; SRT, surgery plus radiotherapy; SC, surgery plus chemotherapy; CRT, chemotherapy plus radiotherapy; SCRT, surgery plus chemotherapy plus radiotherapy; NR, not reported.

\section{Host factors}

Age. With respect to younger individuals ( $<50$ years), the HR for those in the seventh decade of life was $1.3(\mathrm{HR}=1.31$; 95\% CI, 1.19-1.45; $\mathrm{P}=0.00)$. The disease-specific hazards for patients in their 70's were $1.7(\mathrm{HR}=1.69 ; 95 \% \mathrm{CI}, 1.62-1.77$; $\mathrm{P}=0.00)$. A similar pattern was seen with overall survival. There was no evidence of heterogeneity in any of the subgroups (Fig. 2).

Sex. The HR for males was calculated to be 1.1 ( $\mathrm{HR}=1.11$; 95\% CI, 0.93-1.31; $\mathrm{P}=0.26)$. The value was similar for OS $(\mathrm{HR}=1.12 ; 95 \% \mathrm{CI}, 1.03-1.23 ; \mathrm{P}=0.01)$. No statistical heterogeneity was found $\left(\mathrm{I}^{2}=32.14\right)$.

Ethnicity. Pooled HR, with non-Hispanic white Caucasians as reference, was computed for patients with African, Asian/Pacific Island, and other (including white Hispanic, Native American and Mestizos) ancestries. Compared to Caucasian individuals, the hazard to overall survival for non-Caucasians as a whole was $\sim 1.4(\mathrm{HR}=1.39,95 \% \mathrm{CI}$, 1.06-1.82; $\mathrm{P}=0.02)$. Apart from the overall death risk, ethnicity of the affected per se did not have seem to be a major influence on survival (Table II).

Comorbidities and 'High-risk' lifestyle. Having one or more major comorbidities showed a weak correlation to increased risk in all-cause mortality $(\mathrm{HR}=1.43,95 \% \mathrm{CI}$, 1.01-2.04; $\mathrm{P}=0.04)$. On the other hand, the mode of life traditionally considered 'high-risk'-e.g., sedentariness, obesity, smoking-was found to be a significant threat to neither disease-specific $(\mathrm{HR}=1.41,95 \% \mathrm{CI}, 0.98-2.03 ; \mathrm{P}=0.07)$ nor overall $(\mathrm{HR}=1.24,95 \% \mathrm{CI}, 0.98-1.56 ; \mathrm{P}=0.14)$ survival.

\section{Tumour factors}

Cutaneous melanoma. The relative lethality of MM vs. CM was $2.25(\mathrm{HR}=2.27,95 \% \mathrm{CI}, 1.96-2.62 ; \mathrm{P}=0.00)$. No significant heterogeneity was detected across the studies $\left(\mathrm{I}^{2}=26.41\right.$; Fig. 3).

Location. A primary lesion originating within the sino-nasal (SN) cavity was found to be 1.4 times more deadly compared to other locations $(\mathrm{HR}=1.44 ; 95 \% \mathrm{CI}, 1.28-1.63 ; \mathrm{P}=0.00)$. The HR for OS was nearly $2.0(\mathrm{HR}=1.93$; 95\% CI, 1.59-2.33; $\mathrm{P}=0.00)$. Head and neck lesions (MMHN) as a whole showed an HR of $1.4(\mathrm{HR}=1.35 ; 95 \% \mathrm{CI}, 1.02-1.79 ; \mathrm{P}=0.00)$ for overall survival.

Multifocal disease. MM is a devastating cancer partly because of its tendency to arise from multiple foci. The associated disease-specific death risk was nearly 3.0 $(\mathrm{HR}=2.95$; 95\% CI, 2.72-3.19; $\mathrm{P}=0.00)$.

Clinical staging $(M M H N)$. The TNM staging system, developed by the American Joint Committee on Cancer (AJCC), is one of the most widely accepted standards for MMHN staging and conventionally the most accurate predictor of survival. T4 disease (T4a and T4b) was 2.4 times more fatal than T3 tumours (95\% CI, 1.75-2.98; $\mathrm{P}=0.00)$. Meanwhile, N1 disease had an HR of 2.0 compared to N0 ( $\mathrm{HR}=1.90 ; 95 \% \mathrm{CI}$, 1.62-2.23; $\mathrm{P}=0.00)$. For metastatic diseases $(\mathrm{M} 1)$, the HR was $3.2(\mathrm{HR}=3.17$; 95\% CI, 2.72-3.70; $\mathrm{P}=0.00$; Fig. 4).

Clinical features/Macro-morphology. Elevated lactate dehydrogenase (LDH) level was associated with the greatest HR for disease-specific survival ( $\mathrm{HR}=2.06$; 95\% CI, 1.56-2.72; $\mathrm{P}=0.00)$. Higher performance score (PS) was correlated with increased risk for $\mathrm{OS}(\mathrm{HR}=1.71 ; 95 \% \mathrm{CI}, 1.32-2.21 ; \mathrm{P}=0.00)$. 
Table II. Hazard ratios for non-Caucasian ethnicities.

\begin{tabular}{|c|c|c|c|c|c|c|c|}
\hline Ethnicity comparison & Survival & No. of studies & Pooled HR & $95 \% \mathrm{CI}$ & Z-value & P-value & $\mathrm{I}^{2}$ \\
\hline Non-Caucasian vs. Caucasian & DSS & 5 & 1.12 & $1.05-1.20$ & 3.354 & 0.001 & 0.0001 \\
\hline Non-Caucasian vs. Caucasian & OS & 3 & 1.39 & $1.06-1.82$ & 2.358 & 0.018 & 0.0001 \\
\hline Afro-American vs. Caucasian & DSS & 6 & 1.13 & $0.95-1.34$ & 1.421 & 0.155 & 4.451 \\
\hline API vs. Caucasian & DSS & 2 & 1.09 & $0.80-1.49$ & 0.563 & 0.574 & 91.47 \\
\hline
\end{tabular}

HR, hazard ratio; CI, confidence interval; DSS, disease-specific survival; OS, overall survival; API, Asian and Pacific Islander.

Table III. Hazard ratios for clinical/macro-morphological features.

\begin{tabular}{llccrrrr}
\hline Feature comparison & Survival & No. of studies & Pooled HR & 95\% CI & Z-value & P-value & I \\
\hline Elevated LDH vs. WNL & DSS & 4 & 2.06 & $1.56-2.72$ & 5.104 & 0.001 & 0.001 \\
PS>1 vs. PS<0 & OS & 4 & 1.71 & $1.32-2.21$ & 4.112 & 0.001 & 0.001 \\
Ulceration vs. no ulceration & DSS & 3 & 1.32 & $0.91-1.90$ & 1.465 & 0.143 & 6.401 \\
Ulceration vs. no ulceration & OS & 4 & 1.44 & $1.04-2.01$ & 2.191 & 0.215 & 32.95 \\
Pigmentation vs. no pigmentation & OS & 3 & 0.93 & $0.70-1.25$ & 0.464 & 0.642 & 0.001 \\
Necrosis vs. no necrosis & DSS & 2 & 1.29 & $0.96-1.73$ & 1.708 & 0.088 & 0.001 \\
Necrosis vs. no necrosis & OS & 2 & 0.96 & $0.55-1.68$ & 0.013 & 0.989 & 72.12 \\
\hline
\end{tabular}

LDH, lactate dehydrogenase; PS, performance score HR, hazard ratio; CI, confidence interval; DSS, disease-specific survival; OS, overall survival.

\section{Age (DSS)}

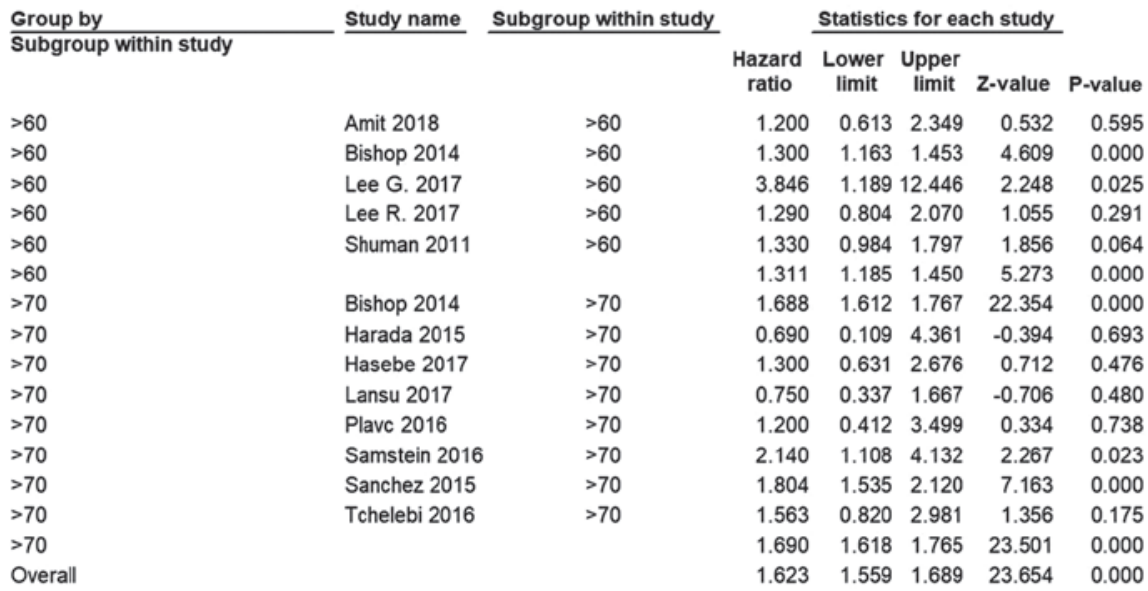

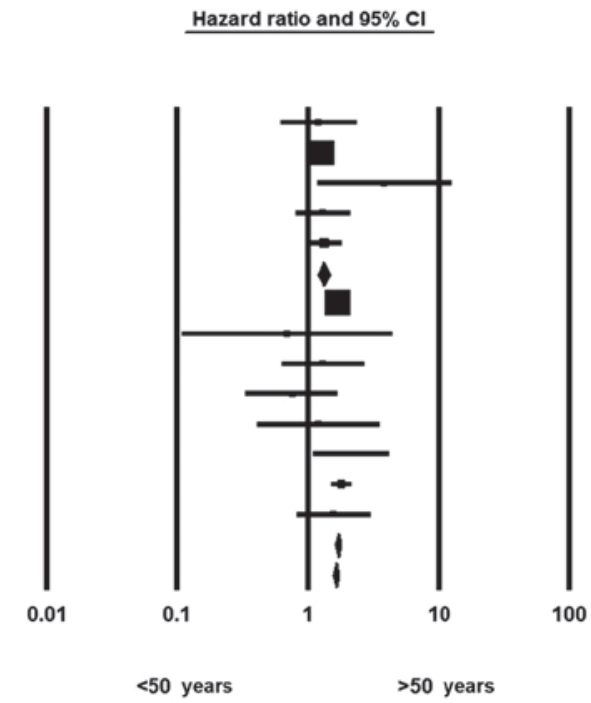

Figure 2. Forest plots for advanced age. DSS, disease-specific survival; CI, confidence interval.

Ulceration of primary lesions was also linked to unfavourable OS. The verdict on pigmentation ( $\mathrm{HR}=0.87$; 95\% CI, 0.66-1.15; $\mathrm{P}=0.34$ ), necrosis, and nodularity of primary tumours was inconclusive (Table III).

Microscopic features. Margin status was the most important micro-morphological determinant of survival. The HR attributed to margin positivity was nearly $2.0(\mathrm{HR}=1.85$; 95\% CI, 1.34-2.54; $\mathrm{P}=0.00)$. The effect of perineural invasion (PNI) and lympho-vascular invasion (LVI) was not statistically significant. Meanwhile, Breslow thickness, depth of invasion, and mitotic count did not seem to play a significant role in either terms of survival (Table IV).

\section{Treatment factors}

Extent of treatment. Radical operation was found to amplify overall death risk by $2.5(\mathrm{HR}=2.61 ; 95 \% \mathrm{CI}, 2.04-3.34$; 
Table IV. Hazard ratios for microscopic features.

\begin{tabular}{|c|c|c|c|c|c|c|c|}
\hline Feature comparison & Survival & No. of studies & Pooled HR & $95 \% \mathrm{CI}$ & Z-value & P-value & $\mathrm{I}^{2}$ \\
\hline (+) Margin vs. (-) margin & DSS & 10 & 1.85 & $1.34-2.54$ & 3.759 & 0.001 & 23.84 \\
\hline (+) Margin vs. (-) margin & OS & 10 & 1.59 & $1.21-2.08$ & 3.365 & 0.001 & 44.22 \\
\hline Breslow $>1 \mathrm{~mm}$ vs. Breslow $<1 \mathrm{~mm}$ & DSS & 6 & 1.07 & $0.99-1.19$ & 1.755 & 0.079 & 29.63 \\
\hline Breslow $>1 \mathrm{~mm}$ vs. Breslow $<1 \mathrm{~mm}$ & OS & 3 & 1.07 & $0.99-1.17$ & 1.621 & 0.105 & 11.23 \\
\hline Invasion $>2 \mathrm{~mm}$ vs. invasion $<2 \mathrm{~mm}$ & DSS & 3 & 2.02 & $0.68-6.03$ & 1.259 & 0.208 & 81.02 \\
\hline Invasion $>2 \mathrm{~mm}$ vs. invasion $<2 \mathrm{~mm}$ & OS & 4 & 2.02 & $1.26-0.23$ & 2.913 & 0.004 & 0.001 \\
\hline Mitosis (+) vs. mitosis (-) & DSS & 4 & 1.09 & $1.03-1.15$ & 2.875 & 0.004 & 0.001 \\
\hline Mitosis (+) vs. mitosis (-) & OS & 4 & 1.06 & $1.01-1.12$ & 2.405 & 0.016 & 0.001 \\
\hline PNI vs. PNI (-) & DSS & 2 & 2.08 & $0.97-4.4$ & 1.884 & 0.06 & 42.65 \\
\hline Lymphovascular invasion vs. no invasion & DSS & 3 & 1.24 & $0.94-1.64$ & 1.537 & 0.124 & 0.001 \\
\hline Epithelioid type vs. non-epithelioid & DSS & 3 & 1.29 & $0.94-1.78$ & 1.561 & 0.118 & 0.001 \\
\hline
\end{tabular}

PNI, perineural invasion; HR, hazard ratio; CI, confidence interval; DSS, disease-specific survival; OS, overall survival.

\begin{tabular}{|c|c|c|c|c|c|c|c|c|c|}
\hline \multirow{3}{*}{ Study name } & \multicolumn{8}{|c|}{ MM vs. CM (DSS) } & \\
\hline & \multicolumn{4}{|c|}{ Statistics for each study } & \multirow[b]{2}{*}{ P-value } & \multicolumn{3}{|c|}{ Hazard ratio and $95 \% \mathrm{Cl}$} & \\
\hline & $\begin{array}{c}\text { Hazard } \\
\text { ratio }\end{array}$ & $\begin{array}{l}\text { Lower } \\
\text { limit }\end{array}$ & $\begin{array}{l}\text { Upper } \\
\text { limit }\end{array}$ & Z-value & & & & & \\
\hline Bishop 2014 & 2.440 & 2.039 & 2.919 & 9.750 & 0.000 & & & & \\
\hline Hughes 2013 & 1.100 & 0.573 & 2.111 & 0.286 & 0.774 & & & & \\
\hline Jang 2014 & 2.155 & 1.550 & 2.996 & 4.567 & 0.000 & & & 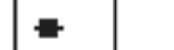 & \\
\hline Kong 2015 & 1.784 & 0.946 & 3.365 & 1.788 & 0.074 & & & 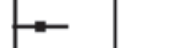 & \\
\hline Oba 2011 & 1.840 & 0.581 & 5.829 & 1.037 & 0.300 & & & & \\
\hline \multirow[t]{3}{*}{ Yi 2011} & 2.988 & 1.534 & 5.821 & 3.217 & 0.001 & & & $\rightarrow$ & \\
\hline & 2.266 & 1.962 & 2.618 & 11.128 & 0.000 & & & $\uparrow$ & \\
\hline & & & & & & 0.01 & 0.1 & 10 & 100 \\
\hline
\end{tabular}

Figure 3. Forest plots for the lethality of mucosal melanoma vs. cutaneous melanoma (DSS). MM, mucosal melanoma; CM, cutaneous melanoma; DSS, disease-specific survival; CI, confidence interval.

$\mathrm{P}=0.00$ ); When surgery was the sole modality of treatment, it was associated with a significant risk elevation in both terms of survival (HR of 1.72 and 2.21, respectively). Conversely, when any modality but surgery was used, similar increase in mortality was observed. For therapeutic regimen consisted entirely of chemotherapy, the attributed risk in mortality was around 1.5. Meanwhile, radiotherapy (RT) apparently carried the least detriment to patient survival as monotherapy.

The value of lymphadenectomy for primary tumours in the cephalo-cervical subsite was dubious $(\mathrm{HR}=0.86 ; 95 \% \mathrm{CI}$, 0.73-1.02; $\mathrm{P}=0.07)$. Likewise, endoscopic resection showed neither inferior nor superior results compared to the more traditional approach in terms of survival benefit $(\mathrm{P}=0.83$ and 0.68 , respectively; Table V).

Adjuvant therapy. Adjuvant chemotherapy was found to reduce both disease-specific and overall death by some 30 percent. The therapeutic regimen included cisplatin/tamoxifen, dacarbazine (DTIC), and interferon- $\gamma($ INF- $\gamma)$. RT, while also significantly effective, tended to be somewhat less efficacious (HR=0.84; 95\% CI, 0.82-0.86; $\mathrm{P}=0.01$; Fig. 5).
Immunotherapy. Immunotherapy, usually involving PD-1 (programmed death protein-1), immune checkpoint inhibitors (e.g., CTLA-4), or a combination of the two, was shown to more effective for MM than CM. The pooled HR was 0.49 (95\% CI, 0.37-0.65; P=0.00; Fig. 6) for overall survival. No inter-study heterogeneity was found across the studies $\left(\mathrm{I}^{2}=0.00\right)$.

\section{Discussion}

The present meta-analysis had aimed to provide an updated review on various aspects of MM, with data from the most recent studies. The genetic and molecular underpinning behind the distinctive biologic behaviour is believed to stem from amplification of c-Kit (11), a receptor tyrosine kinase (RTK). In contrast, b-Raf and n-Ras mutations are infrequent in MM. This oncogenic mutation profile is reminiscent of the acral lentiginous subtype of CM (ALM). Quite fittingly, ALM shares several characteristics with $\mathrm{MM}$ in common, namely i) infrequency (1-2\% of all CM), ii) delayed detection and hence worse prognosis, and iii) relative preponderance in non-Caucasian ethnic groups. 
A

\begin{tabular}{|c|c|c|c|c|c|}
\hline \multirow[t]{2}{*}{ Study name } & \multicolumn{5}{|c|}{ Statistics for each study } \\
\hline & $\begin{array}{c}\text { Hazard } \\
\text { ratio }\end{array}$ & $\begin{array}{l}\text { Lower } \\
\text { limit }\end{array}$ & $\begin{array}{c}\text { Upper } \\
\text { limit }\end{array}$ & Z-value & P-valu \\
\hline Abugideiri 2016 & 3.830 & 1.118 & 13.124 & 2.137 & 0.033 \\
\hline Amit 2018 & 1.290 & 0.329 & 5.051 & 0.366 & 0.715 \\
\hline Benlyazid 2010 & 2.460 & 1.466 & 4.128 & 3.408 & 0.00 \\
\hline Harada 2016 & 2.370 & 0.429 & 13.081 & 0.990 & 0.32 \\
\hline Hasebe 2017 & 1.250 & 0.241 & 6.475 & 0.266 & 0.79 \\
\hline Lansu 2017 & 1.460 & 0.639 & 3.335 & 0.898 & 0.36 \\
\hline Lee R 2016 & 14.400 & 4.601 & 45.064 & 4.582 & 0.00 \\
\hline Samstein 2016 & 2.128 & 1.346 & 3.365 & 3.230 & 0.00 \\
\hline Shuman 2011 & 4.970 & 1.827 & 13.519 & 3.141 & 0.00 \\
\hline Tchelebi 2016 & 1.220 & 0.550 & 2.707 & 0.489 & 0.62 \\
\hline \multirow[t]{2}{*}{ Wang 2013} & 2.590 & 1.768 & 3.795 & 4.882 & 0.00 \\
\hline & 2.409 & 1.947 & 2.980 & 8.093 & 0.00 \\
\hline
\end{tabular}

\section{Hazard ratio and $95 \% \mathrm{Cl}$}

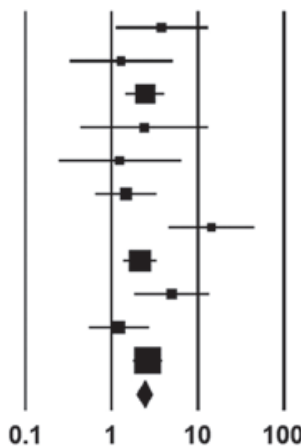

T3 T4

B

\section{N Classification (DSS)}

\begin{tabular}{lrrrrrr} 
Study name & \multicolumn{5}{c}{ Statistics for each study } \\
\cline { 3 - 6 } & & $\begin{array}{c}\text { Hazard } \\
\text { ratio }\end{array}$ & $\begin{array}{c}\text { Lower } \\
\text { limit }\end{array}$ & $\begin{array}{c}\text { Upper } \\
\text { limit }\end{array}$ & Z-value & P-value \\
Ajmani 2017 & 2.450 & 1.009 & 5.952 & 1.979 & 0.048 \\
Amit 2018 & 1.170 & 0.479 & 2.856 & 0.345 & 0.730 \\
Chiu 1996 & 1.400 & 0.942 & 2.081 & 1.663 & 0.096 \\
Ciarrocchi 2016 & 2.630 & 0.405 & 17.096 & 1.013 & 0.311 \\
Harada 2016 & 13.300 & 0.751 & 235.456 & 1.765 & 0.078 \\
Jethanamest 2011 & 1.590 & 1.157 & 2.184 & 2.863 & 0.004 \\
Kirschner 2013 & 1.980 & 1.132 & 3.465 & 2.393 & 0.017 \\
Pandey 2002 & 2.500 & 0.865 & 7.222 & 1.693 & 0.090 \\
Sanchez 2015 & 2.470 & 1.884 & 3.238 & 6.549 & 0.000 \\
Shuman 2011 & 4.440 & 1.679 & 11.742 & 3.004 & 0.003 \\
Tchelebi 2016 & 1.030 & 0.494 & 2.147 & 0.079 & 0.937 \\
& 1.903 & 1.624 & 2.229 & 7.960 & 0.000
\end{tabular}

Hazard ratio and $95 \% \mathrm{Cl}$
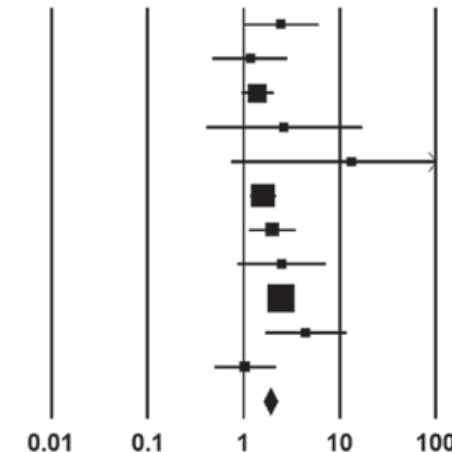

No

N1

C

M Classification (DSS)

Study name

Statistics for each study

Hazard ratio and $95 \% \mathrm{Cl}$

$\begin{array}{ccccc}\begin{array}{c}\text { Hazard } \\ \text { ratio }\end{array} & \begin{array}{c}\text { Lower } \\ \text { limit }\end{array} & \begin{array}{c}\text { Upper } \\ \text { limit }\end{array} & \text { Z-value } & \text { P-value } \\ 3.320 & 1.596 & 6.906 & 3.211 & 0.001 \\ 3.070 & 1.656 & 5.691 & 3.562 & 0.000 \\ 2.800 & 1.721 & 4.556 & 4.146 & 0.000 \\ 2.090 & 0.843 & 5.182 & 1.591 & 0.112 \\ 2.540 & 1.063 & 6.070 & 2.097 & 0.036 \\ 4.550 & 2.920 & 7.091 & 6.694 & 0.000 \\ 2.690 & 1.658 & 4.365 & 4.007 & 0.000 \\ 4.760 & 1.340 & 16.907 & 2.413 & 0.016 \\ 2.972 & 2.259 & 3.910 & 7.786 & 0.000 \\ 15.670 & 3.743 & 65.602 & 3.767 & 0.000 \\ 4.428 & 1.453 & 13.495 & 2.617 & 0.009 \\ 3.400 & 0.986 & 11.729 & 1.937 & 0.053 \\ 1.780 & 1.066 & 2.973 & 2.203 & 0.028 \\ 3.025 & 2.584 & 3.541 & 13.765 & 0.000\end{array}$

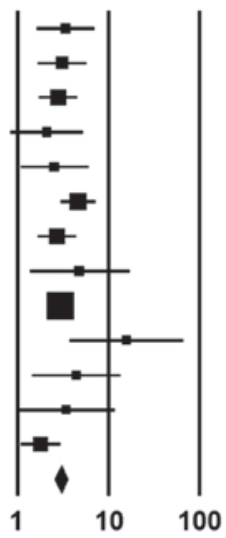

M1

Figure 4. Forest plots for TNM staging (DSS): (A) T4 vs. T3 disease, (B) N1 vs. N0 disease, and (C) M1 vs. M0 disease. DSS, disease specific survival; CI, confidence interval; TNM, tumor-node-metastasis. 
A

\begin{tabular}{lccccr} 
& \multicolumn{5}{c}{ Statistics for each study } \\
\cline { 3 - 6 } & $\begin{array}{l}\text { Hazard } \\
\text { ratio }\end{array}$ & $\begin{array}{c}\text { Lower } \\
\text { limit }\end{array}$ & $\begin{array}{c}\text { Upper } \\
\text { limit }\end{array}$ & Z-value & P-value \\
Ahn 2010 & 0.240 & 0.064 & 0.907 & -2.105 & 0.035 \\
Ajmani 2017 & 0.880 & 0.568 & 1.364 & -0.571 & 0.568 \\
Heppt 2017 & 0.720 & 0.519 & 0.998 & -1.969 & 0.049 \\
Koto 2017 & 0.619 & 0.408 & 0.939 & -2.259 & 0.024 \\
Kuk 2016 & 0.490 & 0.122 & 1.970 & -1.005 & 0.315 \\
Lee G 2017 & 0.140 & 0.017 & 1.131 & -1.844 & 0.065 \\
Sun 2014 & 0.495 & 0.260 & 0.943 & -2.139 & 0.032 \\
& 0.665 & 0.542 & 0.815 & -3.928 & 0.000
\end{tabular}

B

Study name

Amit 2018
Benlyazid 2010
Ciarrocchi 2016
Kirschner 2013
Konuthula 2017
Lansu 2018
Lee R 2017
Schmidt 2017

Hazard ratio and $95 \% \mathrm{Cl}$

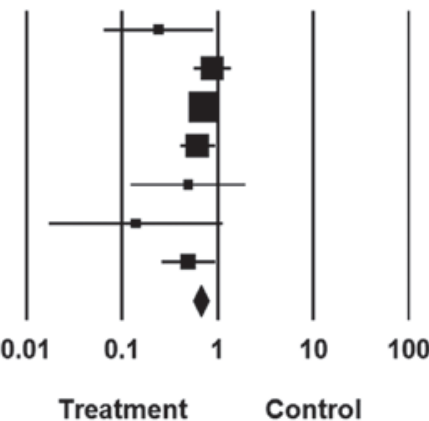

Adj. Radiotherapy (DSS)

Statistics for each study

Lower Upper

ratio limit limit Z-value $P$-value

$\begin{array}{lllll}0.310 & 0.087 & 1.103 & -1.808 & 0.071\end{array}$

$\begin{array}{lllll}0.850 & 0.513 & 1.408 & -0.631 & 0.528\end{array}$

$\begin{array}{lllll}0.877 & 0.324 & 2.374 & -0.258 & 0.796\end{array}$

$\begin{array}{lllll}0.920 & 0.671 & 1.262 & -0.518 & 0.605\end{array}$

$\begin{array}{lllll}0.671 & 0.285 & 1.578 & -0.915 & 0.360\end{array}$

$\begin{array}{lllll}0.820 & 0.301 & 2.236 & -0.388 & 0.698\end{array}$

$\begin{array}{lllll}0.570 & 0.291 & 1.115 & -1.642 & 0.101\end{array}$

$\begin{array}{lllll}0.990 & 0.851 & 1.152 & -0.130 & 0.896\end{array}$

$\begin{array}{lllll}0.928 & 0.819 & 1.052 & -1.168 & 0.243\end{array}$

\section{Hazard ratio and $95 \% \mathrm{Cl}$}

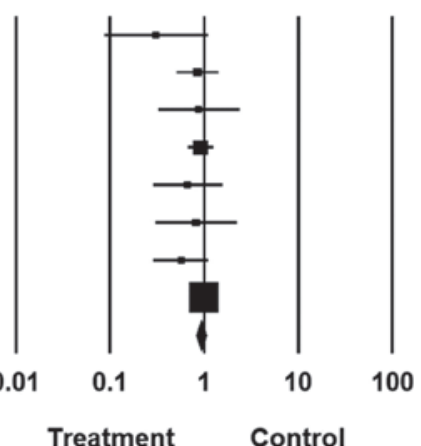

Figure 5. Forest plots for adjuvant therapy (DSS): (A) Chemotherapy and (B) radiation therapy. DSS, disease-specific survival; CI, confidence interval; Adj., adjuvant.

Immunotherapy (OS)

\begin{tabular}{lccccc} 
Study name & & \multicolumn{5}{c}{ Statistics for each study } \\
\cline { 3 - 6 } & $\begin{array}{c}\text { Hazard } \\
\text { ratio }\end{array}$ & $\begin{array}{c}\text { Lower } \\
\text { limit }\end{array}$ & $\begin{array}{c}\text { Upper } \\
\text { limit }\end{array}$ & Z-value & P-value \\
D'Angelo 2016 & 0.503 & 0.349 & 0.725 & -3.677 & 0.000 \\
Schaefer 2017 & 0.620 & 0.310 & 1.240 & -1.352 & 0.176 \\
Nakamura 2018 & 0.520 & 0.241 & 1.123 & -1.664 & 0.096 \\
Wen 2017 & 0.310 & 0.141 & 0.683 & -2.905 & 0.004 \\
& 0.492 & 0.372 & 0.650 & -4.978 & 0.000
\end{tabular}

\section{Hazard ratio and $95 \% \mathrm{Cl}$}

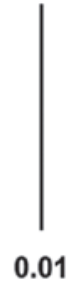

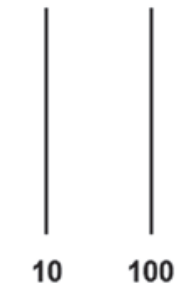

Control

Figure 6. Forest plots for immunotherapy (OS): CI, confidence interval; OS, overall survival.

Although what is known about MM pales in comparison to the cutaneous disease, a few generalities can be drawn from our analysis: in the authors' estimation, MM was two-and-a-quarter times more life-threatening than CM. As a whole, the influence of the 'host factors' was not imposing; one pattern that stood out was advanced age. The median age of onset for MM is higher than CM, at 67 years (vs. 55 years for CM). The death risk in this age group was more than 1.5, compared to the younger cohort ( $<50$ years), which might partially account for the higher mortality. While the incidence tends to be higher and the prognosis grimmer for male melanoma patients in general, MM is an exception; it is reasonably well established that MM shows predilection for females (12). Moreover, there seemed to be no respect of sexes with MM when it comes to mortality, although 
Table V. Hazard ratios for different modalities of treatment.

\begin{tabular}{lcccrrrr}
\hline Modality comparison & Survival & No. of studies & Pooled HR & 95\% CI & Z-value & P-value & $I^{2}$ \\
\hline Radical op. vs. Conservative Tx & OS & 5 & 2.61 & $2.04-3.34$ & 15.079 & 0.001 & 55.35 \\
Op. alone vs. SC/SRT & DSS & 11 & 1.78 & $1.55-2.05$ & 8.192 & 0.001 & 30.85 \\
RT alone vs. SRT & DSS & 5 & 1.29 & $1.08-1.54$ & 2.831 & 0.005 & 19.37 \\
RT alone vs. SRT & OS & 4 & 1.52 & $1.35-1.70$ & 7.087 & 0.001 & 26.97 \\
\hline
\end{tabular}

Op., operation; RT, radiotherapy; SC, surgery plus chemotherapy; SRT, surgery plus radiotherapy; HR, hazard ratio; CI, confidence interval; DSS, disease-specific survival; OS, overall survival.

male individuals may be at a slight disadvantage as far as overall survival is concerned. MM is also peculiar from ethnic perspectives because the higher proportion of non-Caucasian patients (especially African and Asian races) (13) is higher. This point is underlined by the fact that $40 \%$ of the referenced studies came from regions where the indigenous population is not of white Caucasian ancestry. Nevertheless, racial disparities did not appear to be a major deciding factor in MM-specific mortality. The higher all-cause mortality for non-white cohorts may point to either supposedly superior overall quality of care in Western facilities, or a legitimate, ethno-genetic differences in the ability of the body system to cope with the cancer or mount anti-tumour immune defence against. The fact that undesirable health-related behaviours played negligible role in survival may be one indication that the intrinsic cancer behaviour wields an overriding influence above other variables.

Mucosal melanoma of the head and neck (MMHN), cited as the most common location of MM occurrence overall, also carried the worst prognosis. Tumours in the paranasal sinuses (PNS)-maxillary and ethmoid, etc.-predisposed the individuals to significantly higher disease-specific and overall mortality, with the latter perhaps reflecting the inaccessibility of the subsite, rendering it all the more unfeasible to carry out effective surgical manoeuvres. Tumour thickness would normally be one of objective prognosticators for solid organ cancers. That said, the usefulness of the AJCC clinical stageing system in CM cannot be readily engrafted into mucosal patients, the reason for which is questionable validity of tumour thickness as a prognostic index (14). This notion has been backed by the authors' findings, that neither thickness nor depth of invasion is a significant determinant of survival (Table IV).

Although surgery constitutes the backbone of management strategy in many cases, radical excision seems to be a poor choice of treatment for the considerable morbidity and added mortality associated. Any mono-modality therapy was shown to increase death risk by at least 1.5 . For inoperable cases, immunotherapeutic regimen, usually consisting of combination of CTLA-4 and PD-1 inhibitors (e.g., nivolumab and ipilimumab), may be the most rational option. Also, both chemotherapy and radiotherapy were found to be survival-benefitting adjuvant modalities. However, as of now, there is no clearly established formula for specific combination of for chemotherapeutic agents and anti-tumour biologics ('cocktails').

The current study was hampered by a few limitations. The validity of disease-specific survival (DSS), the primary measure of effect sizes, is grounded on the premise of the reported cause of death being accurate. This inherent risk can potentially be a limiting factor with cancers such as MM, in which the high lethality can often obscure the true cause of death. In addition, all but two of the included studies came out after the year 2010. This is mainly due to the rarity of the disease, with many studies taking several decades to complete.

In summing up, mucosal melanoma is a highly malignant entity that is difficult to detect, treat, and even study. It is accentuated by an oncogenic profile that is at odds with the more prevalent cutaneous disease. Microscopic frequency, coupled with air of pessimism surrounding the gross ineffectuality of conventional arsenal, may have pushed it into relative obscurity and disinterest. Nonetheless, a body of recent evidence indicates its incidence is on the rise $(15,16)$, and may well be on its way to becoming a force to be reckoned with. Further studies, elaborating on the oncogenic pathways and driver mutations, are needed to improve the overall outlook of this fearsome cancer, especially now that the era of three P's-precision, personalized, and preventive oncology-is looming over the horizon.

\section{Acknowledgements}

Not applicable.

\section{Funding}

This work was supported by Konyang University Myunggok Research Fund of 16.

\section{Availability of data and materials}

The datasets used and/or analyzed during the current study are available from the corresponding author on reasonable request.

\section{Authors' contributions}

$\mathrm{HMH}$ and KGL designed and conducted the study. $\mathrm{HJH}$ and KGL produced the manuscript. WC, KGL, HJH, HMH, SHC and KBM performed the statistical analysis. All authors read and approved the final manuscript.

\section{Ethics approval and consent to participate}

Not applicable. 


\section{Patient consent for publication}

Not applicable.

\section{Competing interests}

The authors declare that they have no competing interests.

\section{References}

1. Patrick RJ, Fenske NA and Messina JL: Primary mucosal melanoma. J Am Acad Dermatol 56: 828-834, 2007.

2. Tacastacas JD, Bray J, Cohen YK, Arbesman J, Kim J, Koon HB, Honda K, Cooper KD and Gerstenblith MR: Update on primary mucosal melanoma. J Am Acad Dermatol 71: 366-375, 2014.

3. Gru AA, Becker N, Dehner LP and Pfeifer JD: Mucosal melanoma: Correlation of clinicopathologic, prognostic, and molecular features. Melanoma Res 24: 360-370, 2014.

4. Thierauf J, Veit JA, Affolter A, Bergmann C, Grünow J, Laban S, Lennerz JK, Grünmüller L, Mauch C, Plinkert PK, et al: Identification and clinical relevance of PD-L1 expression in primary mucosal malignant melanoma of the head and neck Melanoma Res 25: 503-509, 2015.

5. Ragnarsson-Olding BK, Karsberg S, Platz A and Ringborg UK: Mutations in the TP53 gene in human malignant melanomas derived from sun-exposed skin and unexposed mucosal membranes. Melanoma Res 12: 453-463, 2002.

6. Zhu W, Li S, Zou B, Liu H and Wang S: Expressions and clinical significance of HER4 and CD44 in sinonasal mucosal malignant melanoma. Melanoma Res 28: 105-110, 2018.

7. Kim SY, Kim SN, Hahn HJ, Lee YW, Choe YB and Ahn KJ: Metaanalysis of BRAF mutations and clinicopathologic characteristics in primary melanoma. J Am Acad Dermatol 72: 1036-46. e2, 2015.

8. Tierney JF, Stewart LA, Ghersi D, Burdett S and Sydes MR: Practical methods for incorporating summary time-to-event data into meta-analysis. Trials 8: 16, 2007.

9. Stroup DF, Berlin JA, Morton SC, Olkin I, Williamson GD, Rennie D, Moher D, Becker BJ, Sipe TA and Thacker SB: Meta-analysis of observational studies in epidemiology: A proposal for reporting. Meta-analysis Of Observational Studies in Epidemiology (MOOSE) group. JAMA 283: 2008-2012, 2000

10. Moher D, Liberati A, Tetzlaff J and Altman DG; PRISMA Group: Preferred reporting items for systematic reviews and meta-analyses: The PRISMA statement. PLoS Med 6: e1000097, 2009.

11. Ohashi A, Funasaka Y, Ueda M and Ichihashi M: c-KIT receptor expression in cutaneous malignant melanoma and benign melanotic naevi. Melanoma Res 6: 25-30, 1996.

12. Postow MA, Hamid O and Carvajal RD: Mucosal melanoma: Pathogenesis, clinical behavior, and management. Curr Oncol Rep 14: 441-448, 2012.

13. Altieri L, Wong MK, Peng DH and Cockburn M: Mucosal melanomas in the racially diverse population of California. J Am Acad Dermatol 76: 250-257, 2017.

14. Luna-Ortiz K, Aguilar-Romero M, Villavicencio-Valencia V, Zepeda-Castilla E, Vidrio-Morgado H, Peteuil N and MosquedaTaylor A: Comparative study between two different staging systems for mucosal melanomas of the Head and Neck. Med Oral Patol Oral Cir Bucal 21: e425-e430, 2016.

15. Youssef D, Vasani S, Marquess J and Cervin A: Rising incidence of head and neck mucosal melanoma in Australia. J Laryngol Otol 131: S25-S28, 2017.

16. Marcus DM, Marcus RP, Prabhu RS, Owonikoko TK, Lawson DH, Switchenko J and Beitler JJ: Rising incidence of mucosal melanoma of the head and neck in the United States J Skin Cancer 2012: 231693, 2012.

17. Abugideiri M, Patel K, Switchenko JM, Magliocca K, Buchwald ZS, Delgaudio J, Beitler JJ and Khan MK: Adjuvant radiation therapy improved local control for primary mucosal melanoma of the head and neck. Int J Radiation Oncol 96: E384-E385, 2016

18. Ahn HJ, Na II, Park YH, Cho SY, Lee BC, Lee GH, Koh JS, Lee YS, Shim YS, Kim YK, et al: Role of adjuvant chemotherapy in malignant mucosal melanoma of the head and neck. Oral Oncol 46: 607-611, 2010.
19. Aiempanakit K, Chiratikarnwong K, Auepemkiate S and Sriplung H: Clinicopathologic characteristics and survival outcomes of primary mucosal melanomas: A 10-year retrospective analysis from a single tertiary medical center in Thailand. Zhonghua Pifuke Yixue Zazhi xxx: 1-3, 2018.

20. Ajmani GS, Liederbach E, Kyrillos A, Wang CH, Pinto JM and Bhayani MK: Adjuvant radiation and survival following surgical resection of sinonasal melanoma. Am J Otolaryngol 38: 663-667, 2017.

21. Amit M, Tam S, Abdelmeguid AS, Roberts DB, Raza SM, Su SY, Kupferman ME, DeMonte F and Hanna EY: Approaches to regional lymph node metastasis in patients with head and neck mucosal melanoma. Cancer 124: 514-520, 2018.

22. D'Angelo SP, Larkin J, Sosman JA, Lebbé C, Brady B, Neyns B, Schmidt H, Hassel JC, Hodi FS, Lorigan P, et al: Efficacy and safety of nivolumab alone or in combination with ipilimumab in patients with mucosal melanoma: A pooled analysis. J Clin Oncol 35: 226-235, 2017.

23. Benlyazid A, Thariat J, Temam S, Malard O, Florescu C, Choussy O, Makeieff M, Poissonnet G, Penel N, Righini C, et al: Postoperative radiotherapy in head and neck mucosal melanoma: A GETTEC study. Arch Otolaryngol Head Neck Surg 136: 1219-1225, 2010

24. Bishop KD and Olszewski AJ: Epidemiology and survival outcomes of ocular and mucosal melanomas: A population-based analysis. Int J Cancer 134: 2961-2971, 2014.

25. Chiu NT and Weinstock MA: Melanoma of oronasal mucosa. Population-based analysis of occurrence and mortality. Arch Otolaryngol Head Neck Surg 122: 985-988, 1996.

26. Ciarrocchi A, Pietroletti R, Carlei F and Amicucci G: Extensive surgery and lymphadenectomy do not improve survival in primary melanoma of the anorectum: Results from analysis of a large database (SEER). Colorectal Dis 19: 158-164, 2017.

27. Ercelep O, Topcu TO, Bayoglu IV, Ekinci AS, Koca S, Kavgaci H, Ozcelik M, Alacacioglu A, Uzunoglu S, Bozkurt O, et al: Retrospective multicenter evaluation of patients diagnosed with mucosal melanoma: A study of Anatolian Society of Medical Oncology. Tumour Biol 37: 12033-12038, 2016.

28. Harada K, Mine S, Yamada K, Shigaki H, Oya S, Baba H and Watanabe M: Long-term outcome of esophagectomy for primary malignant melanoma of the esophagus: A single-institute retrospective analysis. Dis Esophagus 29: 314-319, 2016.

29. Hasebe M, Yoshikawa K, Nishii R, Kawaguchi K, Kamada T and Hamada Y: Usefulness of 11C-methionine-PET for predicting the efficacy of carbon ion radiation therapy for head and neck mucosal malignant melanoma. Int J Oral Maxillofac Surg 46: 1220-1228, 2017

30. Heinzelmann-Schwarz VA, Nixdorf S, Valadan M, Diczbalis M, Olivier J, Otton G, Fedier A, Hacker NF and Scurry JP: A clinicopathological review of 33 patients with vulvar melanoma identifies c-KIT as a prognostic marker. Int J Mol Med 33: 784-794, 2014.

31. Heppt MV, Roesch A, Weide B, Gutzmer R, Meier F, Loquai C, Kähler KC, Gesierich A, Meissner M, von Bubnoff D, et al: Prognostic factors and treatment outcomes in 444 patients with mucosal melanoma. Eur J Cancer 81: 36-44, 2017.

32. Hughes MC, Wright A, Barbour A, Thomas J, Smithers BM, Green AC and Khosrotehrani K: Patients undergoing lymphadenectomy for stage III melanomas of known or unknown primary site do not differ in outcome. Int J Cancer 133: 3000-3007, 2013.

33. Jang HS, Kim JH, Park KH, Lee JS, Bae JM, Oh BH, Rha SY, Roh MR and Chung KY: Comparison of melanoma subtypes among Korean patients by morphologic features and ultraviolet exposure. Ann Dermatol 26: 485-490, 2014.

34. Kang X, Zeng Y, Liang J, Li J, Ren D, Chai L, Sun Z, Yu S, Wu X, Han W, et al: Aberrations and clinical significance of BRAF in malignant melanoma: A series of 60 cases in Chinese Uyghur. Medicine (Baltimore) 97: e9509, 2018.

35. Khan MN, Kanumuri VV, Raikundalia MD, Vazquez A, Govindaraj S, Baredes S and Eloy JA: Sinonasal melanoma: Survival and prognostic implications based on site of involvement. Int Forum Allergy Rhinol 4: 151-155, 2014.

36. Kirchoff DD, Deutsch GB, Foshag LJ, Lee JH, Sim MS and Faries MB: Evolving Therapeutic Strategies in Mucosal Melanoma Have Not Improved Survival Over Five Decades. Am Surg 82: 1-5, 2016.

37. Kirschner AN, Kidd EA, Dewees T and Perkins SM: Treatment approach and outcomes of vaginal melanoma. Int J Gynecol Cancer 23: 1484-1489, 2013. 
38. Kong Y, Si L, Li Y, Wu X, Xu X, Dai J, Tang H, Ma M, Chi Z, Sheng X, et al: Analysis of mTOR Gene Aberrations in Melanoma Patients and Evaluation of Their Sensitivity to PI3K-AKT-mTOR Pathway Inhibitors. Clin Cancer Res 22: 1018-1027, 2016.

39. Konuthula N, Khan MN, Parasher A, Del Signore A, Genden EM, Govindaraj S and Iloreta AM: The presentation and outcomes of mucosal melanoma in 695 patients. Int Forum Allergy Rhinol 7: 99-105, 2017.

40. Koto M, Demizu Y, Saitoh JI, Suefuji H, Tsuji H, Okimoto T, Ohno T, Shioyama Y, Takagi R, Nemoto K, et al; Japan Carbon-Ion Radiation Oncology Study Group: Multicenter study of carbon-ion radiation therapy for mucosal melanoma of the head and neck: Subanalysis of the Japan Carbon-Ion Radiation Oncology Study Group (J-CROS) Study (1402 HN). Int J Radiat Oncol Biol Phys 97: 1054-1060, 2017.

41. Kuk SK, Won CH, Lee WJ, Shin WJ, Yoon HJ, Hong SD, Hong SP and Lee J: Prognostic significance of nestin in primary malignant melanoma of the oral cavity. Melanoma Res 26: 457-463, 2016.

42. Lansu J, Klop WM, Heemsbergen W, Navran A, Al-Mamgani A, Langendijk JA, Kaanders JH, Terhaard C, Karakullukcu B and Hamming-Vrieze O: Local control in sinonasal malignant melanoma: Comparing conventional to hypofractionated radiotherapy. Head Neck 40: 86-93, 2018

43. Lawaetz M, Birch-Johansen F, Friis S, Eriksen JG, Kiss K, Gade S, Møller-Madsen M, Pourbordbari N and von Buchwald C: Primary mucosal melanoma of the head and neck in Denmark, 1982-2012: Demographic and clinical aspects. A retrospective DAHANCA study. Acta Oncol 55: 1001-1008, 2016.

44. Lee G, Baek CH, Choi NY and Chung MK: The prognostic role of the surgical approach and adjuvant therapy in operable mucosa melanoma of the head and neck. Clin Exp Otorhinolaryngol 10 : 97-103, 2017

45. Lee RJ,Lee SA, Lin T, Lee KK and Christensen RE: Determining the epidemiologic, outcome, and prognostic factors of ora malignant melanoma by using the Surveillance, Epidemiology, and End Results database. J Am Dent Assoc 148: 288-297, 2017.

46. Lombardi D, Bottazzoli M, Turri-Zanoni M,RaffettiE, Villaret AB Morassi ML, Ungari M, Vermi W, Battaglia P, Castelnuovo P, et al: Sinonasal mucosal melanoma: A 12-year experience of 58 cases. Head Neck 38 (Suppl 1): E1737-E1745, 2016.

47. Mücke T, Hölzle F, Kesting MR, Loeffelbein DJ, Robitzky LK, Hohlweg-Majert B, Tannapfel A and Wolff KD: Tumor size and depth in primary malignant melanoma in the oral cavity influences survival. J Oral Maxillofac Surg 67: 1409-1415, 2009.

48. Nakamura Y, Fujisawa Y, Tanaka R, Maruyama H, Ishitsuka Y, Okiyama N, Watanabe R and Fujimoto M: Use of immune checkpoint inhibitors prolonged overall survival in a Japanese population of advanced malignant melanoma patients: Retrospective single institutional study. J Dermatol 45: 1337-1339, 2018.

49. Oba J, Nakahara T, Abe T, Hagihara A, Moroi Y and Furue M: Expression of c-Kit, p-ERK and cyclin D1 in malignant melanoma: An immunohistochemical study and analysis of prognostic value. J Dermatol Sci 62: 116-123, 2011.

50. Pandey M, Mathew A, Iype EM, Sebastian P, Abraham EK and Nair KM: Primary malignant mucosal melanoma of the head and neck region: Pooled analysis of 60 published cases from India and review of literature. Eur J Cancer Prev 11: 3-10, 2002.

51. Pfeil AF, Leiter U, Buettner PG, Eigentler TK, Weide B, Meier F and Garbe C: Melanoma of unknown primary is correctly classified by the AJCC melanoma classification from 2009 Melanoma Res 21: 228-234, 2011.

52. Plavc G, But-Hadžić J, Aničin A, Lanišnik B, Didanović V and Strojan P: Mucosal melanoma of the head and neck: A population-based study from Slovenia, 1985-2013. Radiat Oncol 11: $137,2016$.

53. Roh MR, Gupta S, Park KH, Chung KY, Lauss M, Flaherty KT, Jönsson G, Rha SY and Tsao H: Promoter Methylation of PTEN Is a Significant Prognostic Factor in Melanoma Survival. J Invest Dermatol 136: 1002-1011, 2016.
54. Samstein RM, Carvajal RD, Postow MA, Callahan MK, Shoushtari AN, Patel SG, Lee NY and Barker CA: Localized sinonasal mucosal melanoma: Outcomes and associations with stage, radiotherapy, and positron emission tomography response. Head Neck 38: 1310-1317, 2016.

55. Sanchez A, Rodríguez D, Allard CB, Bechis SK, Sullivan RJ, Boeke CE, Kuppermann D, Cheng JS, Barrisford GW, Preston MA, et al: Primary genitourinary melanoma: Epidemiology and disease-specific survival in a large population-based cohort. Urol Oncol 34: 166.e7-166.e14, 2016.

56. Schaefer T, Satzger I and Gutzmer R: Clinics, prognosis and new therapeutic options in patients with mucosal melanoma: A retrospective analysis of 75 patients. Medicine (Baltimore) 96: e5753, 2017.

57. Schmidt MQ, David J, Yoshida EJ, Scher K, Mita A, Shiao SL, Ho AS and Zumsteg ZS: Predictors of survival in head and neck mucosal melanoma. Oral Oncol 73: 36-42, 2017.

58. Shoushtari AN, Bluth MJ, Goldman DA, Bitas C, Lefkowitz RA, Postow MA, Munhoz RR, Buchar G, Hester RH, Romero JA, et al: Clinical features and response to systemic therapy in a historical cohort of advanced or unresectable mucosal melanoma. Melanoma Res 27: 57-64, 2017.

59. Shuman AG, Light E, Olsen SH, Pynnonen MA, Taylor JM, Johnson TM and Bradford CR: Mucosal melanoma of the head and neck: Predictors of prognosis. Arch Otolaryngol Head Neck Surg 137: 331-337, 2011.

60. Song H, Jing G, Wang L, Guo W and Ren G: Periodic acid-Schiff-positive loops and networks as a prognostic factor in oral mucosal melanoma. Melanoma Res 26: 145-152, 2016.

61. Sun CZ, Li QL, Hu ZD, Jiang YE, Song M and Yang AK: Treatment and prognosis in sinonasal mucosal melanoma: A retrospective analysis of 65 patients from a single cancer center. Head Neck 36: 675-681, 2014.

62. Tchelebi L, Guirguis A and Ashamalla H: Rectal melanoma: Epidemiology, prognosis, and role of adjuvant radiation therapy. J Cancer Res Clin Oncol 142: 2569-2575, 2016.

63. Thariat J, Poissonnet G, Marcy PY, Lattes L, Butori C, Guevara N, Dassonville O, Santini J, Bensadoun RJ and Castillo L: Effect of surgical modality and hypofractionated split-course radiotherapy on local control and survival from sinonasal mucosal melanoma. Clin Oncol (R Coll Radiol) 23: 579-586, 2011.

64. Wang X, Wen W, Wu H, Chen Y, Ren G and Guo W: Heparanase expression correlates with poor survival in oral mucosal melanoma. Med Oncol 30: 633, 2013.

65. Wen X, Ding Y, Li J,Zhao J, Peng R, Li D, Zhu B, Wang Y, Zhang X and Zhang X: The experience of immune checkpoint inhibitors in Chinese patients with metastatic melanoma: A retrospective case series. Cancer Immunol Immunother 66: 1153-1162, 2017.

66. Won TB, Choi KY, Rhee CS, Jin HR, Yi JS, Dhong HJ, Kim SW, Choi JH, Kim JK, Chung YJ, et al: Treatment outcomes of sinonasal malignant melanoma: A Korean multicenter study. Int Forum Allergy Rhinol 5: 950-959, 2015.

67. Yeh JJ, Shia J, Hwu WJ, Busam KJ, Paty PB, Guillem JG, Coit DG, Wong WD and Weiser MR: The role of abdominoperineal resection as surgical therapy for anorectal melanoma. Ann Surg 244: 1012-1017, 2006.

68. Yi JH, Yi SY, Lee HR, Lee SI, Lim DH, Kim JH, Park KW and Lee J: Dacarbazine-based chemotherapy as first-line treatment in noncutaneous metastatic melanoma: Multicenter, retrospective analysis in Asia. Melanoma Res 21: 223-227, 2011.

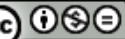

This work is licensed under a Creative Commons Attribution-NonCommercial-NoDerivatives 4.0 International (CC BY-NC-ND 4.0) License. 